\title{
Model Calculation for Viscoelastic and Dielectric Relaxation Functions of Non-Entangled Star Chains with Arm Length Distribution
}

\author{
Hiroshi Watanabe, ${ }^{*}$ Hirotsugu Yoshida, and Tadao Kotaka \\ Department of Macromolecular Science, Faculty of Science, \\ Osaka University, Toyonaka, Osaka 560, Japan
}

(Received September 25, 1989)

\begin{abstract}
Based on Rouse-Ham dynamics, we examined viscoelastic and dielectric relaxation functions of unentangled model star chains of $\mathrm{f}$ arms with and without arm-length distribution. We assumed that each arm possesses the dipole moment aligned in the same direction along the chain contour from the center to the arm end, and thus exhibits the dielectric normal mode process related to center-to-end vector fluctuation of arms. The model calculation suggested that the arm-length distribution influences the mode distribution of the dielectric normal mode relaxation more strongly as compared to that of the viscoelastic relaxation that reflects the orientational anisotropy of the whole segments of the star molecules. This difference is due to the difference in the contribution of the Rouse-Ham eigenfunctions to these two relaxation functions.

KEY WORDS Star Polymer / Viscoelastic Relaxation / Dielectric Relaxation / Dielectric Normal Mode Process / Rouse-Ham Dynamics /
\end{abstract}

The mechanical stress of rubbery and flexible polymer chains is related to the anisotropy of the chain orientation. ${ }^{1-3}$ Viscoelastic spectroscopy has been used extensively for studies of polymer dynamics in this aspect. ${ }^{1}$ On the other hand, for the polymers classified by Stockmayer ${ }^{4}$ as a type-A polymer that has the dipole moment aligned in the same direction parallel along the chain contour, fluctuation of the end-to-end vector induces a change in polarization of the system and thus is dielectrically active. Consequently, dielectric measurements on such polymers give other information on polymer dynamics, i.e., fluctuation of the end-to-end vectors. Polyisoprene (PI) of high cis-content is a typical example of type-A polymers, and we call the dielectric relaxation process due to this global motion of the type-A chains the dielectric normal mode process. ${ }^{5,6}$ Comparing viscoelastic and dielectric behavior

* To whom all correspondence should be addressed. of type-A chains such as cis-PI, we anticipate to obtain more detailed knowledge on the molecular motion of polymer chains.

Generally, the dynamics of polymer chains is strongly influenced through their topological structure. The presence of branches in the chains remarkably changes the behavior of the polymers particularly in the entangled regime. ${ }^{1}$ In this context, the relaxation behavior of star polymers is interesting as an ideal model of branched polymers. Recently we examined viscoelastic behavior of 4-arm polystyrenes blended with monodisperse linear polystyrenes. ${ }^{7}$ We also prepared 6-arm star cis-polyisoprenes (6S-PI) having nearly identical, narrow distribution arms, which are type-A chains with dipoles converging to (or diverging from) the center of the star from (or to) the arm ends, and examined their dielectric relaxation behavior. ${ }^{8}$ In that study, we found that the 
behavior is considerably different from that of linear chains even in the non-entangled regime $^{8}$ where the Rouse-Ham dynamics ${ }^{9-11}$ prevails.

According to the Rouse-Ham dynamics, ${ }^{9,10}$ if the type-A star chain has monodisperse arms, its dielectric behavior should be exactly the same as that of the linear chain identical to the star arms. The experimental results turned out to be otherwise: the dielectric relaxation mode distribution was much broader for the star chains than that for the linear chains corresponding to the arms. These results suggest the dielectric behavior of type-A star chains should be very sensitive to the armlength distribution. In this paper, we compare theoretically the effects of arm-length distribution on viscoelastic and dielectic quantities of type-A star chains, and discuss the differences in these quantities.

\section{THEORETICAL}

\section{Complex Modulus}

We consider an $f$-arm star chain with the $\beta$ th arm being composed of $N_{\beta}$ segments, and number these segments from $n_{\beta}=0$ (the center of the star) to $n_{\beta}=N_{\beta}$ (the free end of the arm). Figure 1 shows the model. Then let $\boldsymbol{r}_{\beta}\left(n_{\beta}, t\right)$ be the spatial position of the $n_{\beta}$-th segment in the $\beta$-th arm at time $t$.

Based on the theory of rubber elasticity, ${ }^{1-3}$ the stress tensor $\sigma(t)$ due to $v_{\mathrm{S}}$ star chains (per unit volume) is given by

$$
\sigma(t)=v_{\mathrm{S}} \frac{3 k T}{b^{2}} \sum_{\beta=1}^{f} \sum_{N_{\beta}=0}^{N_{\beta}-1}\left\langle\Delta \boldsymbol{r}_{\beta}\left(n_{\beta}, t\right) \Delta \boldsymbol{r}_{\beta}\left(n_{\beta}, t\right)\right\rangle+\boldsymbol{p}
$$

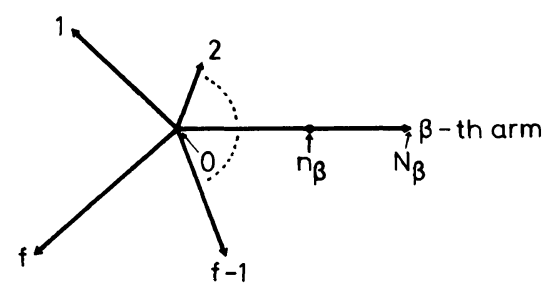

Figure 1. A model f-arm type-A star chain. where $\boldsymbol{p}$ is the isotropic pressure; $\Delta \boldsymbol{r}_{\beta}\left(n_{\beta}, t\right)=$ $\boldsymbol{r}_{\beta}\left(n_{\beta}+1, t\right)-\boldsymbol{r}_{\beta}\left(n_{\beta}, t\right)$, the bond vector connecting the $n_{\beta}$-th and $n_{\beta}+1$-th segments; $b$, the step length of the segment; $k T$, the thermal energy; and $\langle\cdots\rangle$ indicates the average taken over an ensemble of the chains. The tensor $\left\langle\Delta \boldsymbol{r}_{\beta} \Delta \boldsymbol{r}_{\beta}\right\rangle$ represents the anisotropy of orientation of the bond vectors. In the continuous limit, eq 1 becomes

$$
\boldsymbol{\sigma}(t)=v_{\mathrm{S}} \frac{3 k T}{b^{2}} \sum_{\beta=1}^{f} \int_{0}^{N_{\beta}}\left\langle\boldsymbol{u}_{\beta}\left(n_{\beta}, t\right) \boldsymbol{u}_{\beta}\left(n_{\beta}, t\right)\right\rangle \mathrm{d} n_{\beta}+\boldsymbol{p}
$$

where $\boldsymbol{u}_{\beta}=\partial \boldsymbol{r}_{\beta} / \partial n_{\beta}$ is the tangent vector at the $n_{\beta}$-th segment.

The stress relaxation modulus after imposing a step shear strain of the magnitude $\gamma$ (along the $x$-direction) at $t=0$ is obtained from the $x y$ component of $\sigma$ by

$$
G(t)=\sigma_{x y}(t) / \gamma
$$

Then, the complex modulus $G^{*}=G^{\prime}+i G^{\prime \prime}$ is calculated as

$$
G^{*}(\omega)=i \omega \int_{0}^{\infty} G(t) \mathrm{e}^{-i \omega t} \mathrm{~d} t
$$

\section{Complex Dielectric Constant}

In the continuous limit, the (overall) dipole moment $\boldsymbol{P}(t)$ of a type-A star chain at time $t$ is

$$
\boldsymbol{P}(t)=\mu \sum_{\beta=1}^{f} \int_{0}^{N_{\beta}} \boldsymbol{u}_{\beta}\left(n_{\beta}, t\right) \mathrm{d} n_{\beta}
$$

where $\mu$ is the dipole moment per bond vector (cf., Figure 1). The complex dielectric constant $\varepsilon^{*}=\varepsilon^{\prime}-i \varepsilon^{\prime \prime}$ of $v_{\mathrm{S}}$ star chains is obtained from the auto correlation function $V(t)=$ $\langle\boldsymbol{P}(t) \cdot \boldsymbol{P}(0)\rangle /\left\langle\boldsymbol{P}(0)^{2}\right\rangle$ by

$$
\varepsilon^{*}=\varepsilon_{\infty}-v_{\mathrm{S}} \Delta \varepsilon_{\mathrm{S}} \int_{0}^{\infty} \mathrm{d} V(t) / \mathrm{d} t \mathrm{e}^{-i \omega t} \mathrm{~d} t
$$

where $\varepsilon_{\infty}$ is the unrelaxed dielectric constant and $\Delta \varepsilon_{\mathrm{S}}$ is the dielectric relaxation intensity per single star chain. 
Time Evolution of Relaxation Functions

Again in the continuous limit, the RouseHam equation for $\boldsymbol{r}_{\beta}\left(n_{\beta}, t\right)$ is given by ${ }^{9-11}$

$$
\begin{gathered}
\zeta \frac{\partial}{\partial t} \boldsymbol{r}_{\beta}\left(n_{\beta}, t\right)=\kappa \frac{\partial^{2}}{\partial n_{\beta}^{2}} \boldsymbol{r}_{\beta}\left(n_{\beta}, t\right)+\boldsymbol{F}_{\beta}\left(n_{\beta}, t\right) \\
\beta=1, \cdots, f
\end{gathered}
$$

where $\zeta$ is the segmental friction coefficient, $\kappa=3 k T / b^{2}$ and $\boldsymbol{F}_{\beta}$ is the brownian force acting on the $n_{\beta}$-th segment at time $t$. The boundary conditions are $\mathrm{e}^{10.11}$

at the free ends:

$$
\frac{\partial \boldsymbol{r}_{\beta}}{\partial n_{\beta}}=\mathbf{0} \quad \text { at } \quad n_{\beta}=N_{\beta}, \quad \beta=1, \cdots, f
$$

at the center:

$$
\boldsymbol{r}_{\beta}=\boldsymbol{r}_{\alpha} \text { at } n_{\beta}=n_{\alpha}=0, \beta, \alpha=1, \cdots, f
$$

and

$$
\left.\sum_{\beta=1}^{f} \frac{\partial \boldsymbol{r}_{\beta}}{\partial n_{\beta}}\right|_{n_{\beta}=0}=\mathbf{0}
$$

The eigenfunctions for $\boldsymbol{r}_{\beta}$ satisfying eq $7-10$ are classified into two groups: functions, $S_{\beta, k}$ and $C_{\beta, p}$, having and not having a node at the connected end $\left(n_{\beta}=0\right)$, respectively. Using these functions, we can expand $\boldsymbol{r}_{\beta}\left(n_{\beta}, t\right)$ as

$$
\begin{gathered}
\left\{\boldsymbol{r}_{\beta}\left(n_{\beta}, t\right)\right\}=x_{0}(t)\{1\}+\sum_{p} \boldsymbol{x}_{p}(t)\left\{C_{\beta, p}\left(n_{\beta}\right)\right\} \\
+\sum_{k}\left\{z_{\beta, 2 k-1}(t) S_{\beta, 2 k-1}\left(n_{\beta}\right)\right\} \\
C_{\beta, p}=\frac{\cos \lambda_{p}\left(N_{\beta}-n_{\beta}\right)}{\cos \lambda_{p} N_{\beta}}, \quad S_{\beta, 2 k-1}=\sin \frac{2 k-1}{2 N_{\beta}} \pi n_{\beta}
\end{gathered}
$$

Here $\{\cdots\}$ means an array of $f$ quantities (with $\beta=1,2, \cdots, f$ ); $x_{p}$ and $z_{\beta, 2 k-1}$ are the amplitude vectors of eigenfunctions $C_{\beta, p}$ and $S_{\beta, 2 k-1}$, respectively and $\lambda_{p}$ is the eigenvalue for $C_{\beta, p}$ determined by

$$
\sum_{\beta=1}^{f} \tan \lambda_{p} N_{\beta}=0 \quad p=1,2, \cdots
$$

Because of the boundary condition eq 10 , $S_{\beta, 2 k-1}$ functions, and hence $z_{\beta, 2 k-1}$ factors $(\beta=1,2, \cdots, f)$ are not completely independent of each other. Thus, we need to specify independent eignfunctions $S_{\beta, 2 k-1}$ according to the two cases described below, so that the expansion given in eq 11 is a well defined expansion.

Case i) If $j(\geqq 2)$ integers $k_{\beta 1}, k_{\beta 2}, \cdots, k_{\beta j}$ satisfying

$$
\frac{2 k_{\beta 1}-1}{N_{\beta 1}}=\frac{2 k_{\beta 2}-1}{N_{\beta 2}}=\cdots=\frac{2 k_{\beta j}-1}{N_{\beta j}}=\frac{2 k^{*}-1}{N^{*}}
$$

exist, $j(\geqq 2)$ arms, the $\beta_{1}, \beta_{2}, \cdots, \beta_{j}$ th arms, have the degenerate eigenfunctions $S_{\beta_{j}, 2 k_{\beta j}-1}=$ $\sin \left(2 k^{*}-1\right) \pi n_{\beta} / 2 N^{*}$ with the same eigenvalue $\left(2 k^{*}-1\right) \pi / 2 N^{*}$, and the amplitude factors should satisfy

$$
\sum_{i=1}^{j} \boldsymbol{z}_{\boldsymbol{\beta}_{i}, 2 k_{\beta i}-1}=\mathbf{0}
$$

For case i), we have $j-1$ independent eigenfunctions.

Case ii) If no integers $k_{\alpha}$ satisfy

$$
\begin{aligned}
\frac{2 k_{\beta}-1}{N_{\beta}} & =\frac{2 k_{\alpha}-1}{N_{\alpha}}, \\
\alpha & =1, \cdots, \beta-1, \beta+1, \cdots, f
\end{aligned}
$$

for a given integer $k_{\beta}$ (for the $\beta$-th arm), $S_{\beta, 2 k_{\beta}-1}$ is not a proper eigenfunction, and $z_{\beta, 2 k_{\beta}-1}=0$.

For a chain at equilibrium at $t=0$, the average time evolution of $\boldsymbol{z}_{\beta, 2 \boldsymbol{k}_{\beta}-1}$ belonging to case (i) is characterized by the second-order moment

$$
\begin{aligned}
&\left\langle z_{\beta, 2 k_{\beta}-1}(t) z_{\alpha, 2 k_{\alpha}-1}(0)\right\rangle \\
&=\langle\left.z_{\beta, 2 k_{\beta}-1} z_{\alpha, 2 k_{\alpha}-1}\right\rangle_{\mathrm{eq}} \\
& \quad \times \exp \left[-\frac{\kappa}{\zeta}\left(\frac{2 k_{\beta}-1}{2 N_{\beta}}\right)^{2} \pi^{2} t\right]
\end{aligned}
$$

where $\langle\cdots\rangle_{\text {eq }}$ denotes the average at equilibrium. The time evolution of $\boldsymbol{x}_{p}$ is similarly characterized as

$$
\left\langle\boldsymbol{x}_{p}(t) \boldsymbol{x}_{\mathrm{q}}(0)\right\rangle=\left\langle\boldsymbol{x}_{p} \boldsymbol{x}_{q}\right\rangle_{\mathrm{eq}} \exp \left(-\frac{\kappa}{\zeta} \lambda_{p}^{2} t\right)
$$




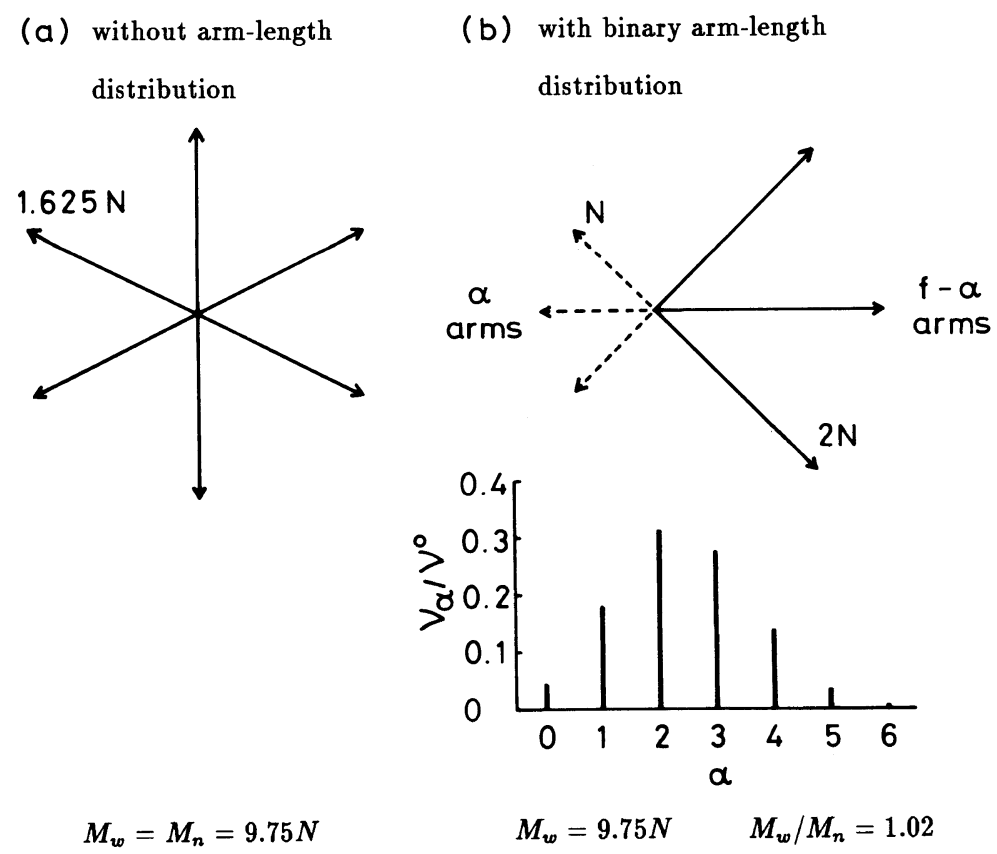

Figure 2. A model $f$-arm star molecule (a) without arm-length distribution and (b) with bimodal arm length distribution.

The averages at equilibrium are evaluated by

$$
\left\langle\boldsymbol{y} \boldsymbol{y}^{\prime}\right\rangle_{\mathrm{eq}}=\frac{\int_{-\infty}^{\infty}\left\{\mathrm{d} \boldsymbol{y}^{\prime \prime}\right\} \boldsymbol{y} \boldsymbol{y}^{\prime} \psi\left(\left\{\boldsymbol{y}^{\prime \prime}\right\}\right)}{\int_{-\infty}^{\infty}\left\{\mathrm{d} \boldsymbol{y}^{\prime \prime}\right\} \psi\left(\left\{\boldsymbol{y}^{\prime \prime}\right\}\right)}
$$

where $y, \boldsymbol{y}^{\prime}$, and $\boldsymbol{y}^{\prime \prime}$ indicate amplitude vectors $\boldsymbol{x}$ and $\boldsymbol{z}$, and the equilibrium distribution function $\psi$ for these vectors is given by

$$
\Psi \propto \exp \left[-(\kappa / 2 k T) \sum_{\beta=1}^{f} \int_{0}^{N_{\beta}}\left(\frac{\partial \boldsymbol{r}_{\beta}}{\partial n_{\beta}}\right)^{2} \mathrm{~d} n_{\beta}\right]
$$

Now, we first focus our attention to a star chain composed of equal arms $\left(N_{1}=N_{2}=\right.$ $\cdots=N_{f}=N$ ) such as shown in Figure 2a. For this case, the eigenfunctions $S_{\beta, 2 k-1}=$ $\sin (2 k-1) \pi n_{\beta} / 2 N$ are degenerate for all arms, leading to the relation

$$
\sum_{\beta=1}^{f} z_{\beta, 2 k-1}=0 \quad \text { for all } k(=1,2, \cdots)
$$

For $C_{\beta, \mathrm{p}}$, the eigenvalue equation is simplified to

$$
\tan \lambda_{p} N=0 ; \lambda_{p}=p \pi / N, p=1,2, \cdots
$$

so that $C_{\beta, p}=\cos (P / N) \pi n_{\beta}$. From eq $19-22$, we find for the star chain with equal arms each composed of $N$ segments,

$$
\begin{gathered}
\sum_{\beta=1}^{f}\left\langle\boldsymbol{x}_{p} z_{\beta, 2 k-1}\right\rangle_{\mathrm{eq}}=\mathbf{0} \\
\left\langle\boldsymbol{x}_{p} \boldsymbol{x}_{q}\right\rangle_{\mathrm{eq}}=\delta_{p q}\left[2 N b^{2} / 3 f \pi^{2} p^{2}\right] \boldsymbol{I} \\
=\delta_{p q}\left[\delta_{\beta \alpha}-(1 / f)\right]\left[8 N b^{2} / 3 \pi^{2}(2 p-1)^{2}\right] \boldsymbol{I}
\end{gathered}
$$$$
\left\langle z_{\beta, 2 p-1} z_{\alpha, 2 q-1}\right\rangle_{\mathrm{eq}}
$$

Here, $\delta_{p q}$ indicates Kronecker's delta, and $\boldsymbol{I}$ is a unit tensor. Using these results, we can calculate the viscoelastic $G(t)$ and dielectric relaxation $V(t)$ functions as follows.

Assuming an affine deformation at $t=0$ for the stress relaxation, ${ }^{2.3}$ we have from eq 11

$$
\begin{aligned}
\boldsymbol{u}_{\beta}\left(n_{\beta}, 0\right) & =\boldsymbol{E} \cdot \boldsymbol{u}_{\beta}^{\mathrm{eq}}\left(n_{\beta}\right) \\
& =(\pi / 2 N) \sum_{k=1}^{\infty}\left[-2 k\left(\boldsymbol{E} \cdot \boldsymbol{x}_{k}^{\mathrm{eq}}\right) \sin \frac{k \pi}{N} n_{\beta}\right.
\end{aligned}
$$




$$
\left.+(2 k-1)\left(\boldsymbol{E} \cdot z_{\beta, 2 k-1}^{\mathrm{eq}}\right) \cos \frac{2 k-1}{2 N} \pi n_{\beta}\right]
$$

where $\boldsymbol{u}_{\beta}{ }^{\text {eq }}$ is a tangent vector at equilibrium; $\boldsymbol{x}_{k}{ }^{\text {eq }}$ and $\boldsymbol{z}_{\beta, 2 k-1}{ }^{\text {eq }}$ are the amplitude vectors at equilibrium characterized by eq $23-25$ and $\boldsymbol{E}$ is a deformation tensor given by

$$
\boldsymbol{E}=\left[\begin{array}{lll}
1 & \gamma & 0 \\
0 & 1 & 0 \\
0 & 0 & 1
\end{array}\right]
$$

From eq $2,3,17,18$, and $22-27, G(t)$ for a star chain with equal arms (identified with the suffices $\mathrm{S}, \mathrm{m}$ ) is calculated by

$$
\begin{aligned}
G_{\mathrm{S}, \mathrm{m}}(t)= & v_{\mathrm{S}} k T\left[\sum_{p=1}^{\infty} \mathrm{e}^{-2 t / \tau(p ; N)}\right. \\
& \left.+(f-1) \sum_{p=1}^{\infty} \mathrm{e}^{-t / 2 \tau(2 p-1 ; N)}\right]
\end{aligned}
$$

where

$$
\tau(p ; N)=\zeta N^{2} b^{2} / 3 \pi^{2} k T p^{2}
$$

Similarly, $V(t)$ for the same star chain is calculated as

$$
V_{\mathrm{S}, \mathrm{m}}(t)=\sum_{p=1}^{\infty}\left(8 / \pi^{2}\right)(2 p-1)^{-2} \mathrm{e}^{-t / \tau(2 p-1 ; N)}
$$

The resulting $G_{\mathrm{s}, \mathrm{m}}(t)$ (eq 28) for the star chain with equal arms is substantially different from $G_{\mathrm{L}, \mathrm{m}}(t)$ given by eq 31 for a monodisperse linear chain identical to the arms

$$
G_{\mathrm{L}, m}(t)=v_{\mathrm{L}} k T \sum_{p=1}^{\infty} \mathrm{e}^{-2 t / \tau(p ; N)}
$$

On the other hand, $V_{\mathrm{s}, \mathrm{m}}(t)$ (eq 30) is exactly the same as $V_{\mathrm{L}, \mathrm{m}}(t)$ of the linear chain.

This difference between $G$ and $V$ for a star chain is related to the difference in the nature of the mechanical and dielectric responses of type-A polymer chains. As can be clearly seen from eq 1 , the mechanical stress measures the sum of the orientation anisotropy of bond vectors $\left\langle\Delta \boldsymbol{r}_{\beta} \Delta \boldsymbol{r}_{\beta}\right\rangle$ of the individual segments.
In other words, $\left\langle\Delta \boldsymbol{r}_{\beta} \Delta \boldsymbol{r}_{\alpha}\right\rangle$ for the different segments $\beta$ and $\alpha(\neq \beta)$ does not contribute to the stress. On the other hand, the auto correlation function

$$
\begin{gathered}
V(t) \propto \sum_{\beta} \sum_{\alpha} \sum_{n_{\beta}} \sum_{n_{\alpha}}\left\langle\Delta \boldsymbol{r}_{\beta}\left(n_{\beta}, t\right) \cdot \Delta \boldsymbol{r}_{\alpha}\left(n_{\alpha}, 0\right)\right\rangle \\
\text { (discrete expression) }
\end{gathered}
$$

involves the orientation correlation between all pairs of bond vectors in the star molecule.

The monodisperse type-A star chain with equal arms examined above has a high symmetry in its architecture and thus, all arms have the same eigenfunctions $S_{\beta, 2 k-1}$, leading to the following relation ( $c f$., eq 21 ),

$$
\begin{gathered}
\sum_{\beta=1}^{f} z_{\beta, 2 k-1} \int_{0}^{N_{\beta}} \frac{\partial S_{\beta, 2 k-1}}{\partial n_{\beta}} \mathrm{d} n_{\beta}=\mathbf{0} \\
\text { for } \quad k=1,2, \cdots
\end{gathered}
$$

so that the overall contribution to $V(t)$ of these eigenfunctions vanishes. Too, the eigenvalue equation for $C_{\beta, p}$ is reduced to a very simple form (eq 22), again because of this high symmetry. These are the reasons why such a star chain exhibits dielectric behavior exactly the same as that of the corresponding linear chain. On the other hand, in general for stars with arm-length distribution, the symmetry is broken so that eq 33 does not hold and $S_{\beta, 2 k-1}$ may contribute to $V(t)$. In addition to this, the eigenvalue equation (eq 13) for $C_{\beta, p}$ gives nondegenerate sequences of $\lambda_{p}$, as we shall see later in eq 38. Thus, we expect $V(t)$ to have broader mode distribution for stars with arm-length distribution than those with equal arms.

For general arm-distribution, it is difficult to solve eq 13 analytically to find an explicit expression for $\lambda_{p}$. It is also difficult to find appropriate $k$ values satisfying eq 14 and giving non-zero $z_{\beta, k-1}$ factors. We thus analyze the dynamics of a model $f$-arm star with a simple arm-length distribution function, and compare their behavior with that of a star with equal arms (Figure 2a).

We consider an ensemble of long and short 
linear chains of length $2 N$ and $N$ with the number-fraction $\phi_{l}$ and $\phi_{s}\left(=1-\phi_{l}\right)$, respectively. Using these chains as precursors, we can make f-arm star chains with arm-length distribution. Further, assuming that the formation of star chains to obey random coupling statistics, we can evaluate the number $v_{\alpha}$ of star chains composed of $\alpha$ short ( $N$-segments) arms and $f-\alpha$ long ( $2 N$-segments) arms by

$$
v_{\alpha}=v^{\mathbf{0}} \frac{f !}{\alpha !(f-\alpha) !} \phi_{s}^{\alpha} \phi_{l}^{f-\alpha}
$$

where

$$
\nu^{\mathrm{o}}\left(=\sum_{\alpha=0}^{f} v_{\alpha}\right)
$$

is the total number of the star chains in unit volume. We refer to such a star chain composed of $\alpha$ short and $f-\alpha$ long arms as a type- $\alpha$ star chain. Figure $2 b$ shows the model star chains with bimodal arms.

On the basis of the Rouse-Ham dynamics, we assume that the motion of a star chain is not correlated with that of the others. Thus, $G(t)$ and $V(t)$ for such an ensemble of the type$\alpha$ star chains $(\alpha=0,1, \cdots, f)$ are simply given by

$$
\begin{gathered}
G(t)=\left[\sum_{\alpha=0}^{f} \sigma_{\alpha}(t)\right]_{x y} / \gamma \\
V(t)=\sum_{\alpha=0}^{f} v_{\alpha}\left\langle\boldsymbol{P}_{\alpha}(t) \cdot \boldsymbol{P}_{\alpha}(0)\right\rangle / \sum_{\alpha=0}^{f} v_{\alpha}\left\langle\boldsymbol{P}_{\alpha}^{2}\right\rangle_{\mathrm{eq}}
\end{gathered}
$$

where the quantities, $\sigma_{\alpha}(t)$ and $v_{\alpha}\left\langle\boldsymbol{P}_{\alpha}(t) \cdot \boldsymbol{P}_{\alpha}(0)\right\rangle$, obtained by eq 2 (with $v_{S}$ being replaced by $v_{\alpha}$ ) and 5 , respectively, indicate the contribution of the type- $\alpha$ chains in the ensemble.

For the type- $\alpha$ star chain we are considering, the eigenvalue equation for $C_{\beta, p}$ reduces to

$$
\alpha \tan \lambda N+(f-\alpha) \tan 2 \lambda N=0
$$

which in turn leads to three sequences of $\lambda_{p}$

$$
\begin{gathered}
\lambda_{p, \alpha}=p \pi / N, p=1,2, \cdots \\
\lambda_{p, \alpha}^{\prime}=\left(p-1+\chi_{\alpha}\right) \pi / N, p=1,2, \cdots \\
\lambda_{p, \alpha}=\left(p-\chi_{\alpha}\right) \pi / N, p=1,2, \cdots
\end{gathered}
$$

with

$$
\chi_{\alpha}=(1 / \pi) \cos ^{-1} \sqrt{\alpha / 2 f}\left(0<\chi_{\alpha}<1 / 2\right)
$$

We also note that the integers satisfying eq 14 are found only for arms of the same length ( $N$ or $2 N$ ) for the present case. This makes the calculation for $V(t)$ much easier, because the relation ( $c f$. , eq 33$)$

$$
\begin{aligned}
& \sum_{\beta}^{\prime} z_{\beta, 2 k-1} \int_{0}^{N_{\beta}} \frac{\partial S_{\beta, 2 k-1}}{\partial n_{\beta}} d n_{\beta}=\mathbf{0} \\
& \sum_{\beta}^{\prime}=\text { the sum for equal arms }
\end{aligned}
$$

still holds for the equal arms so that the overall contribution of the eigenfuctions $S_{\alpha, 2 k-1}$ to $V(t)$ again vanishes.

For the type- $\alpha$ star chain with $\alpha$ short and $f-\alpha$ long arms, the second-order moments of the amplitude vectors at equilibrium are characterized by ( $c f$. , eq 19 and 20)

$$
\begin{aligned}
\left\langle\boldsymbol{x}_{p} \boldsymbol{x}_{q}\right\rangle_{\mathrm{eq}}= & \delta_{p q}\left(2 b^{2} / 3 \lambda_{p}^{2} N\right) \\
& \times\left[\frac{\alpha}{\cos ^{2} N \lambda_{p}}+\frac{2(f-\alpha)}{\cos ^{2} 2 N \lambda_{p}}\right]^{-1} I
\end{aligned}
$$

where $\lambda_{p}\left(=\lambda_{p, \alpha}, \lambda_{p, \alpha}^{\prime}\right.$, or $\left.\lambda^{\prime \prime}{ }_{p, \alpha}\right)$ is given eq $38 \mathrm{a}-$ $38 \mathrm{c}$, and

$$
\begin{aligned}
& \left\langle z_{\beta, 2 k-1} z_{\beta^{\prime}, 2 k^{\prime}-1}\right\rangle_{\mathrm{eq}} \\
& =\delta_{\mathrm{kk}} \cdot\left[8 N^{*} b^{2} / 3 \pi^{2}(2 k-1)^{2}\right]\left[\delta_{\beta \beta^{\prime}}-\left(1 / \alpha^{*}\right)\right] \boldsymbol{I} \\
& \text { for } N_{\beta}=N_{\beta},
\end{aligned}
$$

where $N^{*}=N, \alpha^{*}=\alpha$ for $N_{\beta}=N_{\beta^{\prime}}=N$ and $\alpha \geqq 2$, and $N^{*}=2 N, \alpha^{*}=f-\alpha$ for $N_{\beta}=N_{\beta^{\prime}}=2 N$ and $f-\alpha \geqq 2$.

As we did for stars with equal arms, we obtain $G(t)$ and

$$
\langle\boldsymbol{P}(t) \cdot \boldsymbol{P}(0)\rangle=\sum_{\alpha=0}^{f} \nu_{\alpha}\left\langle\boldsymbol{P}_{\alpha}(t) \cdot \boldsymbol{P}_{\alpha}(0)\right\rangle
$$

for the ensemble of stars with bimodal armdistribution using eq $38-41$ instead of $22-25$ and 33. The results are

$$
G_{\mathrm{S}, \mathbf{b}}(t)=v^{\mathrm{o}} k T \sum_{p=1}^{\infty}\left[\sum_{\alpha=1}^{f-1} \phi_{s}^{\alpha} \phi_{l}^{f-\alpha} \frac{f !}{\alpha !(f-\alpha) !}\right.
$$




$$
\begin{aligned}
& \times\left\{\mathrm{e}^{-2 t / \tau^{\prime}(p ; N ; \alpha)}+\mathrm{e}^{-2 t / \tau^{\prime \prime}(p ; N ; \alpha)}\right\} \\
& +\left(\phi_{l}^{f}+f \phi_{s}-1\right) \mathrm{e}^{-t / 2 \tau(2 p-1 ; N)} \\
& +\left(\phi_{s}^{f}+f \phi_{l}-1\right) \mathrm{e}^{-t / 8 \tau(2 p-1 ; N)} \\
& +\left(1-\phi_{l}^{f}\right) \mathrm{e}^{-2 t / \tau(p ; N)} \\
& \left.+\phi_{l}^{f} \mathrm{e}^{-t / 2 \tau(p ; N)}\right]
\end{aligned}
$$

and

$\langle\boldsymbol{P}(t) \cdot \boldsymbol{P}(0)\rangle$

$$
\begin{aligned}
= & v^{\mathrm{o}} \mu^{2} N b^{2} \sum_{p=1}^{\infty}\left[\sum_{\alpha=1}^{f-1} \phi_{s}^{\alpha} \phi_{l}^{f-\alpha}\right. \\
& \times \frac{f !}{\alpha !(f-\alpha) !}\left\{g_{p, \alpha} \mathrm{e}^{-t / \tau(p ; N)}\right. \\
& \left.+g_{p, \alpha}^{\prime} \mathrm{e}^{-2 t / \tau^{\prime}(p ; N ; \alpha)}+g_{p, \alpha}^{\prime \prime} \mathrm{e}^{-2 t / \tau^{\prime \prime}(p ; N ; \alpha)}\right\} \\
& +\phi_{s}^{f}\left[8 f / \pi^{2}(2 p-1)^{2}\right] \mathrm{e}^{-t / \tau(2 p-1 ; N)} \\
& \left.+\phi_{l}^{f}\left[16 f / \pi^{2}(2 p-1)^{2}\right] \mathrm{e}^{-t / 4 \tau(2 p-1 ; N)}\right]
\end{aligned}
$$

where

$$
\begin{gathered}
\tau^{\prime}(p ; N ; \alpha)=\zeta b^{2} N^{2} /\left[3 \pi^{2} k T\left(p-1+\chi_{\alpha}\right)^{2}\right] \\
p=1,2, \cdots \\
\tau^{\prime \prime}(p ; N ; \alpha)=\zeta b^{2} N^{2} /\left[3 \pi^{2} k T\left(p-\chi_{\alpha}\right)^{2}\right] \\
p=1,2, \cdots \\
g_{p, \alpha}=\left(2 \alpha^{2} / p^{2} \pi^{2}\right)[1-\cos p \pi]^{2} /(2 f-\alpha) \\
g_{p, \alpha}^{\prime}=\left(2 / N^{2} \lambda^{\prime}{ }_{p, \alpha}{ }^{2}\right)\left[\alpha / \cos N \lambda^{\prime}{ }_{p, \alpha}\right. \\
\left.+(f-\alpha) / \cos 2 N \lambda_{p, \alpha}-f\right]^{2}\left[\alpha / \cos ^{2} N \lambda^{\prime}{ }_{p, \alpha}\right. \\
\left.+2(f-\alpha) / \cos { }^{2} 2 N \lambda^{\prime}{ }_{p, \alpha}\right]^{-1} \\
g^{\prime \prime}{ }_{p, \alpha}= \\
+\left(2 / N^{2} \lambda^{\prime \prime}{ }_{p, \alpha}{ }^{2}\right)\left[\alpha / \cos N \lambda^{\prime \prime}{ }_{p, \alpha}\right. \\
\left.+(f-\alpha) / \cos ^{2} 2 N \lambda^{\prime \prime}{ }_{p, \alpha}-f\right]^{2}\left[\alpha / \cos ^{2} N \lambda^{\prime \prime}{ }_{p, \alpha}\right. \\
\left.+2(f-\alpha) / \cos ^{2} 2 N \lambda^{\prime \prime}{ }_{p, \alpha}\right]^{-1}
\end{gathered}
$$

and $\tau(p ; N)$ and $\chi_{\alpha}$ are given in eq 29 and $38 \mathrm{~d}$, respectively.

\section{DISCUSSION}

We now compare the dynamic response of the two model star systems having the same weight-average molecular weight, one being an ensemble of stars with the bimodal arm-length distribution, and the other, an ensemble of monodisperse stars with equal arms ( $c f$., Figure $2 \mathrm{a}$ and $\mathrm{b})$. The number fractions of the short ( $\mathrm{N}-$ ) and long ( $2 \mathrm{~N}$-segments) arms in the former system were $\phi_{s}=2 / 5$ and $\phi_{l}=3 / 5$, giving the polydispersity index of the arms $\left(M_{w} / M_{n}\right)^{\mathrm{arm}}=1.09$, that of the whole chains $\left(M_{w} / M_{n}\right)^{\text {star }}=1.02$, and $M_{w}^{\text {star }}=9.75 N$. Correspondingly, the arm length for the latter system was chosen as $1.625 N$ so that $M_{w}{ }^{\text {star }}=$ $9.75 \mathrm{~N}$.

Figures 3 and 4 compare $G^{\prime \prime}(\omega)$ and $\varepsilon^{\prime \prime}(\omega)$ curves, respectively, for these two model systems of 6 -arm stars $(f=6)$. These quantities are calculated by eq 4 and 6 from $G(t)$ and $V(t)$. We choose and compare $G^{\prime \prime}(\omega)$ and $\varepsilon^{\prime \prime}(\omega)$ because these quantities are equally sensitive to the slow mode distribution, as most clearly seen from the expression based on the mechanical and dielectric relaxation spectra $H(\tau)$ and $g(\tau)$

$$
\begin{aligned}
G^{\prime \prime}(\omega) & =\int_{-\infty}^{\infty} \frac{\omega \tau}{1+\omega \tau} H(\tau) \mathrm{d} \ln \tau \\
\varepsilon^{\prime \prime}(\omega) & =\int_{-\infty}^{\infty} \frac{\omega \tau}{1+\omega \tau} g(\tau) \mathrm{d} \ln \tau
\end{aligned}
$$

(Note that $G^{\prime}$ is much more sensitive and $\varepsilon^{\prime}$ is much less sensitive to the slow mode distribution as compared to $G^{\prime \prime}$ and $\varepsilon^{\prime \prime}$.)

In Figures 3 and 4, we note that the shape of the $G^{\prime \prime}$ curves is almost indistinguishable for stars with bimodal arm-length distribution (eq 42) and those with equal arms (eq 28 with $N$ being replaced by $1.625 N$ ), while the $\varepsilon^{\prime \prime}$ curve is considerably broader for the former (eq 43) than for the latter (eq 31 with $N$ again replaced by $1.625 N$ ). This difference between $G^{\prime \prime}$ and $\varepsilon^{\prime \prime}$ is again attributable to the difference in the contribution of eigenfunctions $S_{\beta, 2 k-1}$ to these quantities.

As mentioned earlier, the overall contribution of $S_{\beta, 2 k-1}$ to $\varepsilon^{\prime \prime}$ (or $V$ ) vanishes and only the $C_{\beta, p}$ contribute to $\varepsilon^{\prime \prime}$ for both equal arm 


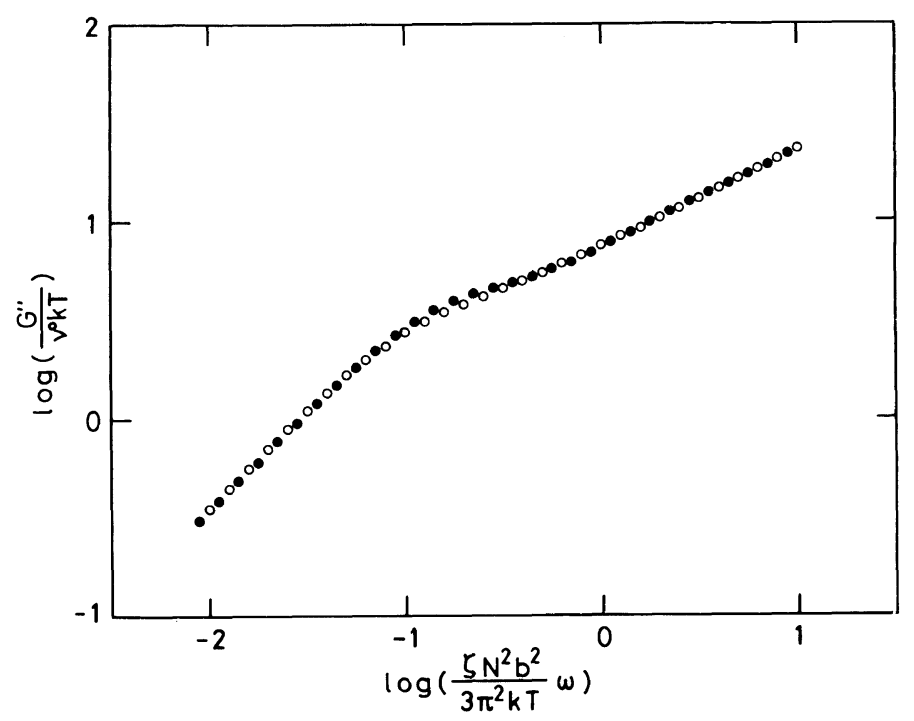

Figure 3. Comparison of $G^{\prime \prime}$ curves for two model stars having the same weight-average molecular weight. $\bigcirc$, stars with bimodal arm-length distribution with $\left(M_{w} / M_{n}\right)^{\mathrm{arm}}=1.09$ and $\left(M_{w} / M_{n}\right)^{\mathrm{star}}=1.02$; monodisperse stars with equal arms.

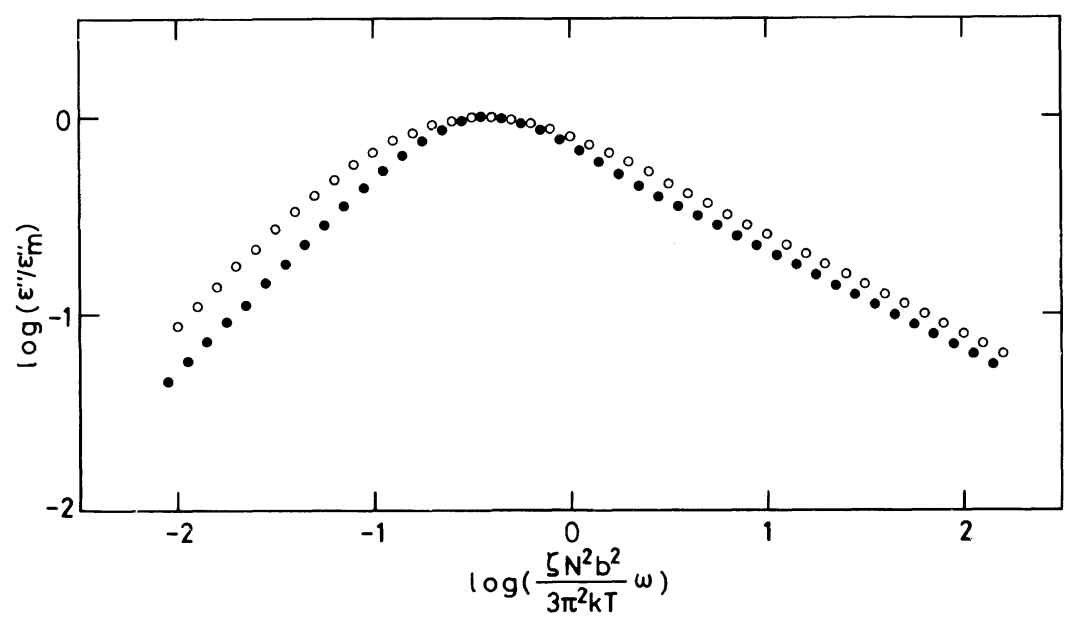

Figure 4. Comparison of shape of $\varepsilon^{\prime \prime}$ curves for the two model stars examined in Figure 3. $\bigcirc$, stars with bimodal arm-distribution; $\odot$, equal arm stars. The two curves are reduced at the peaks for easy comparison.

stars and stars with long and short arms examined here. Thus, the change in the eigenvalue equation for $C_{\beta, p}$ (from eq 22 to 37 ) due to the arm lehgth distribution is totally responsible for the difference in the relaxation mode distribution. On the other hand, $S_{\beta .2 k-1}$ contributes dominantly to the slow relaxation modes for $G^{\prime \prime}$ (or $\left.G(t)\right)$. (Note that the slowest mode for $G^{\prime \prime}$ correspons to $S_{\beta, 1}$.) This is the reason why $\varepsilon^{\prime \prime}$ is significantly different but $G^{\prime \prime}$ is not between the two model systems with $\left(M_{w} / M_{n}\right)^{\text {star }}=1$ and 1.02 examined.

The results shown in Figures 3 and 4 explain qualitatively the experimental observation ${ }^{8}$ 
that the $\varepsilon^{\prime \prime}$ curve in the non-entangled regime is substantially broader for stars than precursor linear chains, both having narrow molecular weight distribution. On the other hand, the steady state compliance of stars and precursors is in agreement with the prediction of the Rouse-Ham theory. ${ }^{12}$ Dielectric quantities appear to be more sensitive to the small distribution of the arm-length as compared to viscoelastic quantities. In this connection, we have to emphasize that more precise knowledge on the arm-length distribution is necessary for an interpretation of $\varepsilon^{\prime \prime}$ data than that for $G^{\prime \prime}$. In other words, even the slightest distribution of the arm length might obscure the interpretation of the $\varepsilon^{\prime \prime}$ data but not necessarily so for the viscoelastic data.

Finally, we wish to point out that real star PI samples may have much broader armlength distribution than that expected for random-coupling model star systems considered here. To obtain model stars, we have assumed random coupling of bimodal precursor linear chains. However, during real star-synthesis via anionic living polymerization of narrow distribution precursors followed by coupling with a 6-functional coupler, the coupling of long precursor chains becomes slower at the later stage of the reaction, because of steric hindrance due to arms having been already coupled. Thus, shorter linear chains may be coupled more easily at the later stages, leading to a broader arm-length distribution. (In fact, the $M_{w} / M_{n}$ ratio (=1.08) for star chains obtained was nearly the same as that of linear precursors. ${ }^{8}$ This contradicts the expectation from the random coupling assumption that should make the $M_{w} / M_{n}$ ratio of the stars narrower than that of the precursors.)

The surprisingly large influence of the armlength distribution on the dielectric normal mode relaxation should lead to $\varepsilon^{\prime \prime}$ curves much broader for the experimentally available stars than that expected for the hypothetical stars obeying the random coupling model.

Acknowledgements. This work was supported in part by a Grant-in-Aid for Scientific Research (No. 60470107) from the Ministry of Education, Science, and Culture of Japan. A support from the Institute of Marcromolecular Research (Osaka University) was also gratefully acknowledged.

\section{REFERENCES}

1. See, for example, J. D. Ferry, "Viscoelastic Properties of Polymers," 3rd ed., Wiley, New York, N.Y., 1980.

2. W. W. Graessley, Adv. Polym. Sci., 47, 67 (1982).

3. M. Doi and S. F. Edwards, "The Theory of Polymer Dynamics," Clarendon Press, Oxford, 1986.

4. W. H. Stockmayer, Pure Appl. Chem., 15, 539 (1969).

5. K. Adachi and T. Kotaka, Macromolecules, 17, 120 (1984); ibid., 18, 466 (1985).

6. Y. Imanishi, K. Adachi, and T. Kotaka, J. Chem. Phys., 89, 7585 (1988).

7. H. Watanabe, H. Yoshida, and T. Kotaka, Macromolecules, 21, 2175 (1988), and the references cited therein.

8. H. Yoshida, K. Adachi, H. Watanabe, and T. Kotaka, Polym. J., 21, 863 (1989).

9. P. E. Rouse, J. Chem. Phys., 21, 1272 (1953).

10. J. S. Ham, J. Chem. Phys., 26, 625 (1957).

11. B. H. Zimm and R. W. Kilb, J. Polym. Sci., 37, 294 (1959).

12. W. W. Graessley and J. Roovers, Macromolecules, 12, 959 (1979). 